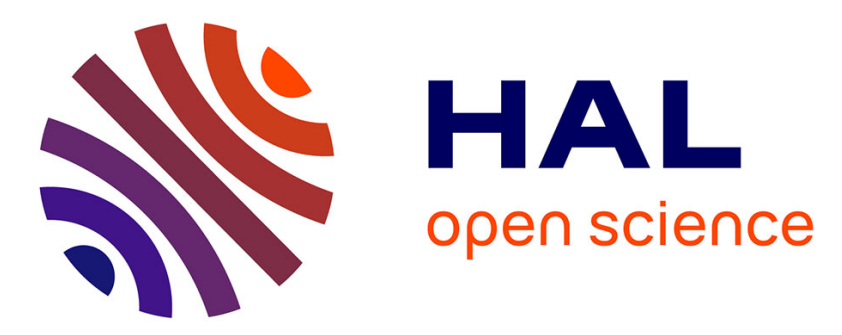

\title{
Charge dynamics in capacitively coupled radio frequency discharges
}

J Schulze, E Schüngel, Z Donkó, U Czarnetzki

\section{To cite this version:}

J Schulze, E Schüngel, Z Donkó, U Czarnetzki. Charge dynamics in capacitively coupled radio frequency discharges. Journal of Physics D: Applied Physics, 2010, 43 (22), pp.225201. 10.1088/0022$3727 / 43 / 22 / 225201$. hal-00569618

\section{HAL Id: hal-00569618 \\ https://hal.science/hal-00569618}

Submitted on 25 Feb 2011

HAL is a multi-disciplinary open access archive for the deposit and dissemination of scientific research documents, whether they are published or not. The documents may come from teaching and research institutions in France or abroad, or from public or private research centers.
L'archive ouverte pluridisciplinaire HAL, est destinée au dépôt et à la diffusion de documents scientifiques de niveau recherche, publiés ou non, émanant des établissements d'enseignement et de recherche français ou étrangers, des laboratoires publics ou privés. 


\title{
Charge dynamics in capacitively coupled radio frequency discharges
}

\author{
J. Schulze ${ }^{1}$, E. Schüngel ${ }^{1}$, Z. Donkó ${ }^{2}$, and U. Czarnetzki ${ }^{1}$ \\ ${ }^{1}$ Institute for Plasma and Atomic Physics, Ruhr-University Bochum, Germany \\ ${ }^{2}$ Research Institute for Solid State Physics and Optics of the Hungarian Academy of \\ Science, Budapest, Hungary \\ E-mail: fjschulze@hotmail.com
}

\begin{abstract}
In a capacitively coupled radio frequency (CCRF) discharge the number of positive and negative charges lost to each electrode must balance within one RF period to ensure a constant total uncompensated charge in the discharge, $Q_{t o t}$, on time average. This balance is the result of a compensation of electron and ion fluxes at each electrode within one RF period. Although $Q_{t o t}$ is constant on temporal average, it is time dependent on time scales shorter than one RF period, since it results from a balance of the typically constant ion flux and the strongly time dependent electron flux at each electrode. Nevertheless, $Q_{t o t}$ is assumed to be constant in various models. Here the dynamics of $Q_{t o t}$ is investigated in a geometrically symmetric CCRF discharge operated in argon at $13.56 \mathrm{MHz}$ and $27.12 \mathrm{MHz}$ with variable phase shift $\theta$ between the driving voltages by a PIC simulation and an analytical model. Via the Electrical Asymmetry Effect (EAE) a variable DC self bias is generated as a function of $\theta$. It is found that $Q_{t o t}$ is not temporally constant within the low frequency period, but fluctuates by about $10 \%$ around its time average value. This modulation is understood by an analytical model. It is demonstrated that this charge dynamics leads to a phase shift of the DC self bias not captured by models neglecting the charge dynamics. This dynamics is not restricted to dual frequency discharges. It is a general phenomenon in all CCRF discharges and can generally be described by the model introduced here. Finally, $Q_{t o t}$ is split into the uncompensated charges in each sheath. The sheath charge dynamics and the self-excitation of non-linear Plasma Series Resonance oscillations of the RF current via the EAE at low pressures of a few Pa are discussed.
\end{abstract}

PACS numbers: 52.20.-j, 52.27.Aj, 52.40.Kh, 52.50.Qt, 52.65.Rr

Submitted to: J. Phys. D: Appl. Phys. 


\section{Introduction}

Capacitively coupled radio frequency (CCRF) discharges are used for a variety of technological applications such as plasma etching and deposition processes. These processes are most important for e.g. semiconductor manufacturing and the production of biocompatible surfaces [1]. For these applications the flux of charged particle species, i.e. electrons and ions, to the surfaces in contact with the plasma and its dynamics are essential. A detailed insight into charge dynamics is required for an optimization of these applications and for understanding fundamental phenomena such as electron heating [1-15] and ion bombardment of surfaces in CCRF discharges [2, 16-18].

In electropositive discharges there is a positive total uncompensated charge in the discharge:

$$
Q_{t o t}=Q_{i}-Q_{e}>0
$$

Here $Q_{i}=e N_{i}$ and $Q_{e}=e N_{e}$, where $N_{i}$ is the total number of positive charges in the discharge, i.e. the number of ions, if all ions carry a single positive charge, and $N_{e}$ is the total number of negative charges in the discharge, i.e. the number of electrons. This uncompensated charge is to a good approximation purely located inside the sheaths [19]. In various models describing CCRF discharges $Q_{t o t}$ or $\sigma_{\text {tot }}=Q_{t o t} / A$, where $A$ is the electrode surface area, is assumed to be temporally constant within the RF period [19$23]$.

Under typical processing conditions positive ions are continuously accelerated towards the electrodes by the time averaged sheath electric fields. Only particularly light ions such as hydrogen or helium ions can follow the fast modulation of the sheath potential at frequencies similar to $13.56 \mathrm{MHz}[2,3]$. The ion flux at each electrode is usually constant within the RF period. However, the electron flux to the electrodes is strongly time modulated, since electrons are lost to an electrode only during the short phases of sheath collapse. During these time intervals the electron flux compensates the ion flux to the respective electrode time integrated over the entire RF period to ensure a constant total uncompensated charge in the discharge on time average, so that the plasma does not continuously charge up. During the rest of the RF period the electron flux to the electrode is zero, since the high sheath potential, $\phi_{s}$, prevents electrons from getting to the electrode during most of the RF period $\left(\phi_{s} \gg k T_{e}\right.$, where $T_{e}$ is the electron temperature). Only for particularly low sheath potentials or very highly energetic electrons, such as secondary electrons, this might no longer be true. Due to this qualitatively known charge dynamics $\sigma_{\text {tot }}$ cannot be temporally constant within the $\mathrm{RF}$ period, since it results from a balance of the temporally constant ion and the strongly time dependent electron flux to each electrode.

In this work the dynamics of $\sigma_{\text {tot }}$ within the RF period in a geometrically symmetric CCRF discharge operated in argon at $13.56 \mathrm{MHz}$ and $27.12 \mathrm{MHz}$ with variable phase shift $\theta$ between the driving voltages is investigated in detail based on PIC simulation and model results. $\sigma_{\text {tot }}$ is found to be time modulated by about $10 \%$ around its time average 
value. This modulation is understood by an analytical model. It is demonstrated that a negligence of this dynamics in models describing CCRF discharges can affect the correct prediction of plasma parameters. As an example the model describing the Electrical Asymmetry Effect (EAE) [2, 19,24-31] in dual frequency discharges is discussed. In this model any charge dynamics is neglected. It is demonstrated that the charge dynamics leads to a phase shift of the DC self bias generated as an almost linear function of the phase shift between the two driving frequencies by the EAE, which has not been observed by the EAE model, but by a PIC simulation before.

Finally, the total uncompensated charge is split into the charges in each sheath. The dynamics of the uncompensated charge in each sheath is investigated in charge phase space. As a result self excited non-linear Plasma Series Resonance (PSR) oscillations of the RF current in geometrically symmetric, but electrically asymmetric CCRF discharges are observed at low pressures of a few Pa.

The paper is structured in the following way: In the second section basics of the PIC simulation used to investigate the charge dynamics and the discharge conditions examined in this work are outlined. In section three the analytical model used to describe the charge dynamics in CCRF discharges is introduced. In the fourth section the simulation results are presented. This section is split into two parts: First, the dynamics of the total uncompensated charge in the discharge is discussed. Second, the dynamics of the charge in each sheath is investigated. Finally, conclusions are drawn in section five.

\section{Particle in Cell simulation}

The simulation used in this work is a one-dimensional $(1 \mathrm{~d} 3 \mathrm{v})$ bounded plasma particlein-cell simulation complemented with a Monte Carlo treatment of collision processes (PIC/MCC). At the planar, parallel and infinite electrodes, electrons are reflected with a probability $\alpha$ (typically $0 \%-20 \%$ ). The secondary electron emission coefficient $\gamma$ is chosen to be $0-0.1$. The neutral gas temperature is taken to be $T_{g}=350 \mathrm{~K}$. Electrode gaps $d$ of $6.7 \mathrm{~cm}$ and $2.5 \mathrm{~cm}$ are used. The discharge is operated as an electrically asymmetric dual frequency discharge $[2,19,24-31]$. The following voltage waveform is applied to the bottom electrode:

$$
\tilde{\phi}(t)=\tilde{\phi}_{0}[\cos (2 \pi f t+\theta)+\cos (4 \pi f t)]
$$

Here $f=13.56 \mathrm{MHz}$ is the applied fundamental radio frequency, $\tilde{\phi}_{0}$ is the identical amplitude of the applied fundamental and second harmonic, and $\theta$ is the fixed, but adjustable phase shift between the driving voltages, which is used to generate a DC self bias $\eta$ as a function of $\theta$ via the $\operatorname{EAE}[2,19,26,27]$. For the sake of simplicity identical voltage amplitudes for both harmonics are used, although a particular choice of different amplitudes is known to allow the generation of a stronger DC self bias [30]. $\eta$ is determined in an iterative way to ensure that the charged particle fluxes to the two electrodes, averaged over one low frequency period, balance. 
The discharge is operated in argon. The cross sections for electron-neutral and ionneutral collision processes are taken from [32-34]. Details of the PIC simulation can be found elsewhere [26, 35-37].

The charge in the sheath per area adjacent to the powered and grounded electrode, $\sigma_{p}$ and $\sigma_{g}$, are defined as:

$$
\begin{aligned}
& \sigma_{p}(t)=e \int_{0}^{s_{p}(t)} n_{i}^{p}(z) d z \\
& \sigma_{g}(t)=e \int_{d-s_{g}(t)}^{d} n_{i}^{g}(z) d z
\end{aligned}
$$

Here $n_{i}^{p, g}(z)$ is the ion density in the respective sheath, that depends on the distance $z$ from the powered electrode. The powered electrode is located at $z=0$ and the grounded electrode is located at $z=d . s_{p, g}(t)$ is the time dependent width of the sheath adjacent to the powered and grounded electrode, respectively. The sheath widths are defined by the following criteria [20]:

$$
\begin{aligned}
& \int_{0}^{s_{p}(t)} n_{e}^{p}(z, t) d z=\int_{s_{p}(t)}^{d / 2}\left[n_{i}^{p}(z)-n_{e}^{p}(z, t)\right] d z \\
& \int_{d-s_{g}(t)}^{d} n_{e}^{g}(z, t) d z=\int_{d / 2}^{d-s_{g}(t)}\left[n_{i}^{g}(z)-n_{e}^{g}(z, t)\right] d z
\end{aligned}
$$

In this work $\sigma_{p, g}(t)$ is calculated from the electric field resulting from the PIC simulation directly adjacent to the respective electrode based on Poisson's equation and assuming an electron density $n_{e}=0$ in the sheath:

$$
\begin{aligned}
& \sigma_{p}(t)=\varepsilon_{0} E(z=0, t) \\
& \sigma_{g}(t)=\varepsilon_{0} E(z=d, t)
\end{aligned}
$$

Here $\varepsilon_{0}$ is the dielectric constant and $E(z, t)$ is the electric field.

Under the assumption that the bulk is quasi-neutral, the total uncompensated charge density in the discharge, $\sigma_{t o t}$, is the sum of the uncompensated charge densities in both sheaths:

$$
\sigma_{t o t}(t)=\sigma_{p}(t)+\sigma_{g}(t)
$$

\section{Analytical model to describe the charge dynamics}

The uncompensated charge density in the discharge, $\sigma_{t o t}$, is positive at all times, since more ions than electrons are located in the plasma. Obviously, the assumption of a temporally constant charge - made in different models of CCRF discharges [19-23] - can only be approximately, however, not exactly true: Ions are continuously lost from the discharge at a constant rate due to the ion fluxes to both electrodes. Only at the short phases of sheath collapse at each of the electrodes electrons are lost and the total positive 


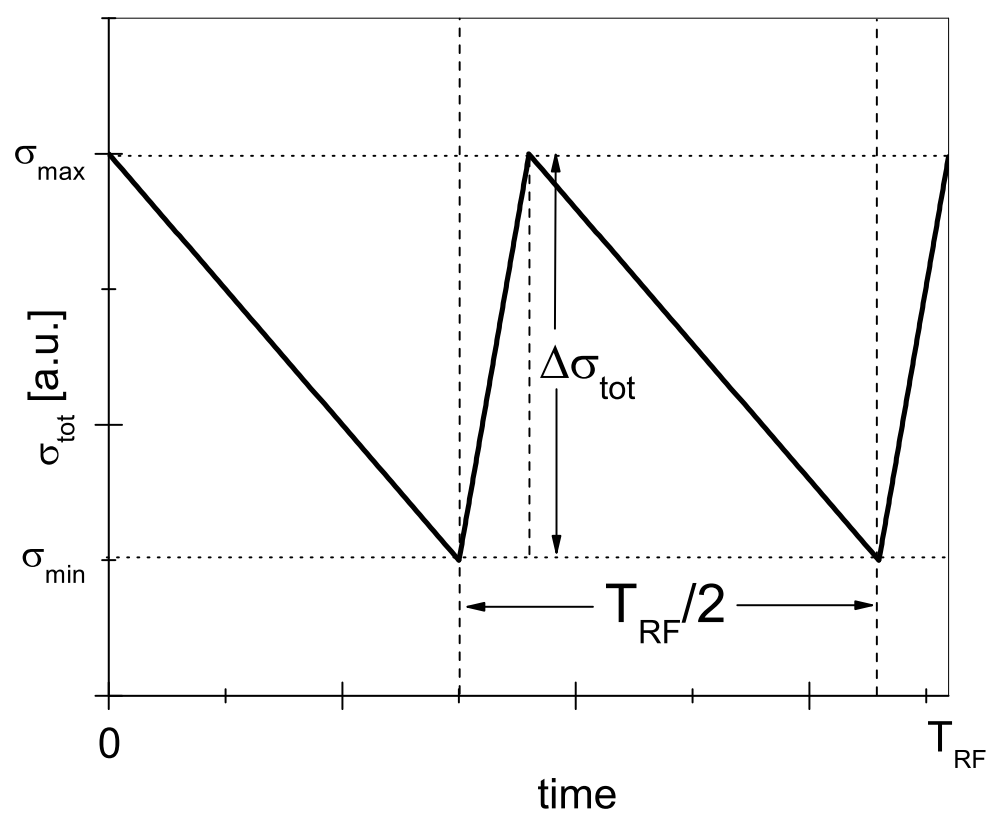

Figure 1. Sketch of the charge dynamics assumed in the frame of the analytical model used to estimate $\Delta \sigma_{\text {tot }}$. One sheath collapse at each electrode within one RF period with equal time intervals $T_{R F} / 2$ in between is assumed. Although shown in the sketch, the short times of sheath collapse, when electrons leave the discharge, are neglected in the model calculations.

charge increases again (see figure 1 for a qualitative sketch of the charge dynamics). The loss rate of $\sigma_{\text {tot }}$ can be estimated based on the following analytical model:

The one dimensional continuity equations for electrons and ions are:

$$
\begin{aligned}
\frac{\partial n_{e}}{\partial t}+\frac{\partial}{\partial z}\left(n_{e} u_{e}\right) & =n_{e} \nu_{i z} \\
\frac{\partial n_{i}}{\partial t}+\frac{\partial}{\partial z}\left(n_{i} u_{i}\right) & =n_{e} \nu_{i z}
\end{aligned}
$$

Here $n_{e}, n_{i}$ is the electron and ion density, respectively, $u_{e}, u_{i}$ is the electron and ion velocity, respectively, and $\nu_{i z}$ is the ionization frequency. Subtraction of equations 10 and 11 yields:

$$
\frac{\partial}{\partial t}\left(n_{i}-n_{e}\right)+\frac{\partial}{\partial z}\left(n_{i} u_{i}-n_{e} u_{e}\right)=0
$$

The short phases of sheath collapse, when electrons compensate the ion losses to one electrode, are neglected in the model calculations. Instead it is assumed, that the ion losses accumulated over half an RF period are compensated instantaneously by the electron losses to one electrode at the phase of sheath collapse. Thus, $n_{e}=0$ is assumed at the electrodes and integration of equation 12 over the entire discharge length $d$ yields:

$$
\frac{\partial \sigma_{t o t}}{\partial t}+\left[\left.\left(n_{i} u_{i}\right)\right|_{d}-\left.\left(n_{i} u_{i}\right)\right|_{0}\right]=0
$$


Under the assumption of equal ion densities $n_{i, e l}$ and absolute values of the ion velocities $u_{i, e l}$ at the electrodes equation 13 yields:

$$
\frac{\partial \sigma_{t o t}}{\partial t}=-2 n_{i, e l} u_{i, e l}
$$

The spatially averaged ion density in the sheath $\bar{n}_{i}$ can be approximated by the ion density at the electrode $\bar{n}_{i} \approx n_{i, e l}[19]$. Assuming one sheath collapse at each electrode with equal time intervals $T_{R F} / 2$ between two sheath collapses, i.e. a single frequency symmetric discharge operated at a frequency $f=1 / T_{R F}$ (see figure 1 ), the order of magnitude of the relative fluctuation of $\sigma_{\text {tot }}$ can be estimated:

$$
\frac{\Delta \sigma_{t o t}}{\bar{\sigma}_{t o t}}=-\frac{u_{i, e l}}{s_{\max }} T_{R F}
$$

Here $\bar{\sigma}_{t o t}$ is the time average value of $\sigma_{t o t}, \Delta \sigma_{t o t}=\sigma_{\max }-\sigma_{\min }$ (see figure 1), $s_{\max }$ is the maximum sheath width of the respective sheath, and $T_{R F}$ is the duration of one $\mathrm{RF}$ period $\left(\bar{\sigma}_{t o t}=s_{\max } \bar{n}_{i}\right)$.

Based on the assumption of a Matrix sheath $s_{\max }$ is given by:

$$
s_{\max }=\sqrt{\frac{2 \varepsilon_{0} \hat{\phi}_{s}}{e \bar{n}_{i}}}
$$

Here $\hat{\phi}_{s}$ is the maximum sheath voltage. Based on the conservation of ion energy in the sheath the ion velocity at the electrode can be calculated from the temporal average of the sheath potential $\bar{\phi}_{s} \approx \hat{\phi}_{s} / 2$ :

$$
u_{i, e l} \approx \sqrt{\frac{e \hat{\phi}_{s}}{m_{i}}}
$$

Substitution of equations 16 and 17 into equation 15 yields the following result for argon, an applied RF frequency of $13.56 \mathrm{MHz}$, and a typical ion density in the sheath of $\bar{n}_{i} \approx 1.1 \times 10^{9} \mathrm{~cm}^{-3}$ taken from the PIC simulation:

$$
\frac{\Delta \sigma_{t o t}}{\bar{\sigma}_{t o t}} \leq \sqrt{2} \pi \frac{\omega_{p i, s}}{\omega_{R F}} \approx 0.1
$$

here $\omega_{p i, s}$ and $\omega_{R F}$ correspond to the ion plasma frequency in the sheath and the applied $\mathrm{RF}$ frequency, respectively. Based on this model the total uncompensated charge in the discharge is expected to fluctuate by about $10 \%$ around its time average value.

\section{Simulation results}

\subsection{Dynamics of the total uncompensated charge in an electrically asymmetric dual} frequency discharge

In this section the dynamics of the total uncompensated charge density, $\sigma_{t o t}$, in a geometrically symmetric electrically asymmetric dual frequency discharge operated at 13.56 MHz and 27.12 MHz with fixed, but adjustable phase shift $\theta$ between the driving 
voltages is investigated by a PIC simulation. Results are compared to the results of the analytical model introduced in the previous section. The discharge is operated in argon at $2.66 \mathrm{~Pa}(20 \mathrm{mTorr})$ and an electrode gap of $6.7 \mathrm{~cm}$ similar to conditions investigated by Godyak et al. experimentally [38] and identical with the discharge conditions investigated by the analytical model of the EAE and the Brinkmann sheath model [19]. $\alpha=0.2$ and $\gamma=0.1$ are used. The voltage waveform defined by equation 2 with $\tilde{\phi}_{0}=315 \mathrm{~V}$ is applied to the bottom electrode.

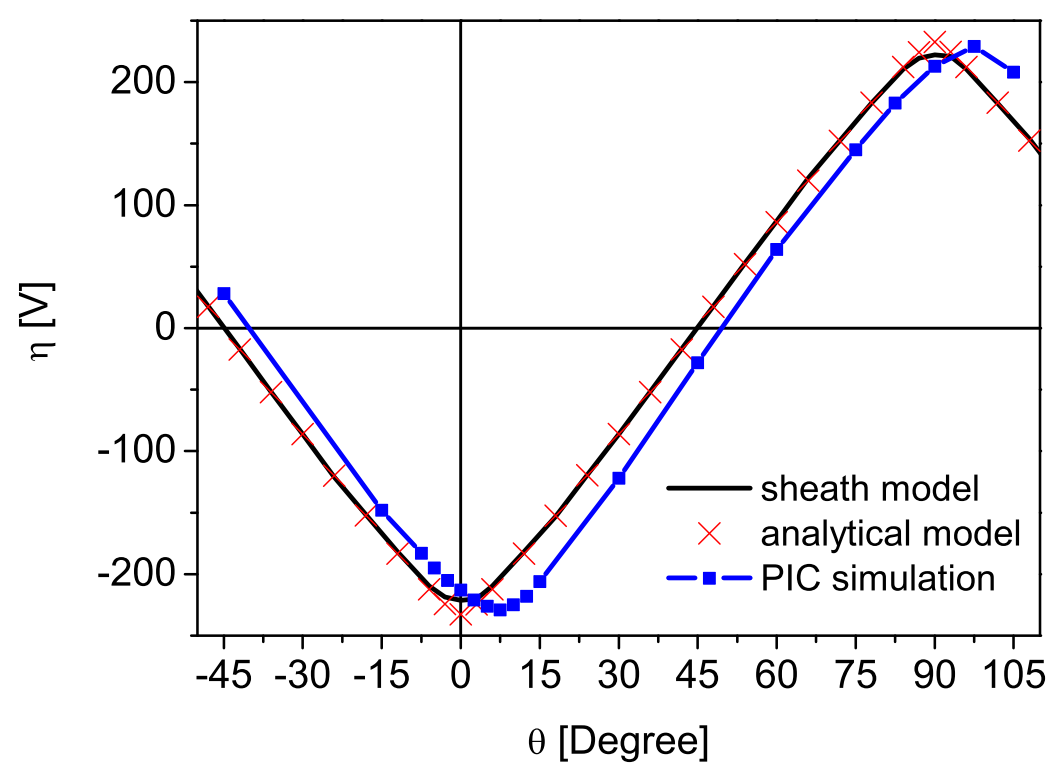

Figure 2. DC self bias $\eta$ calculated by the Brinkmann sheath model (solid black line, [19]), the analytical model (red markers, [19]) and the PIC simulation (blue markers and solid line, [26]) under the same discharge conditions. This plot is taken from reference [26].

Via the EAE a DC self bias $\eta$ is generated as an almost linear function of $\theta$. Figure 2 shows $\eta$ as a function of $\theta$ resulting from the analytical model of the EAE (red markers, [19]), the Brinkmann sheath model (solid black line, [19]), and the PIC simulation (blue markers and solid line, [26]) under identical discharge conditions. A phase shift of about $\Delta \theta \approx 8^{\circ}$ between the analytical model and the Brinkmann sheath model on one side and the PIC simulation on the other side is observed.

In the frame of the analytical model of the EAE (red markers in figure 2) the DC self bias $\eta$ is calculated from the extrema of the applied voltage waveform and the symmetry parameter $\varepsilon[19]$ :

$$
\eta=-\frac{\tilde{\phi}_{m 1}+\varepsilon \tilde{\phi}_{m 2}}{1+\varepsilon}
$$

Here $\tilde{\phi}_{m 1}, \tilde{\phi}_{m 2}$ is the maximum and minimum of equation 2 , respectively. The symmetry 
parameter is defined as:

$$
\varepsilon=\left|\frac{\hat{\phi}_{s g}}{\hat{\phi}_{s p}}\right|=c_{p g}\left(\frac{A_{p}}{A_{g}}\right)^{2} \frac{\bar{n}_{s p}}{\bar{n}_{s g}}\left(\frac{Q_{m g}}{Q_{m p}}\right)^{2}
$$

Here $\hat{\phi}_{s g}, \hat{\phi}_{s p}$ are the maximum sheath voltages, that drop across the sheath at the grounded and powered electrode, respectively. $A_{p}, A_{g}$ are the surface areas of the powered and grounded electrode, respectively, and $\bar{n}_{s p}, \bar{n}_{s g}$ are the mean ion densities in the respective sheath. $c_{p g}=I_{s g} / I_{s p}$ is the ratio of the sheath integrals such as defined in reference [19]. Generally, $c_{p g}$ is close to unity for all phase angles $\theta$. For the discharge conditions investigated here $c_{p g} \approx 1$ was explicitly verified by a PIC simulation [26]. Thus, for the geometrically symmetric $\left(A_{p}=A_{g}\right)$ discharge investigated here:

$$
\varepsilon=\frac{\bar{n}_{s p}}{\bar{n}_{s g}}\left(\frac{Q_{m g}}{Q_{m p}}\right)^{2}
$$

\section{Grounded electrode}

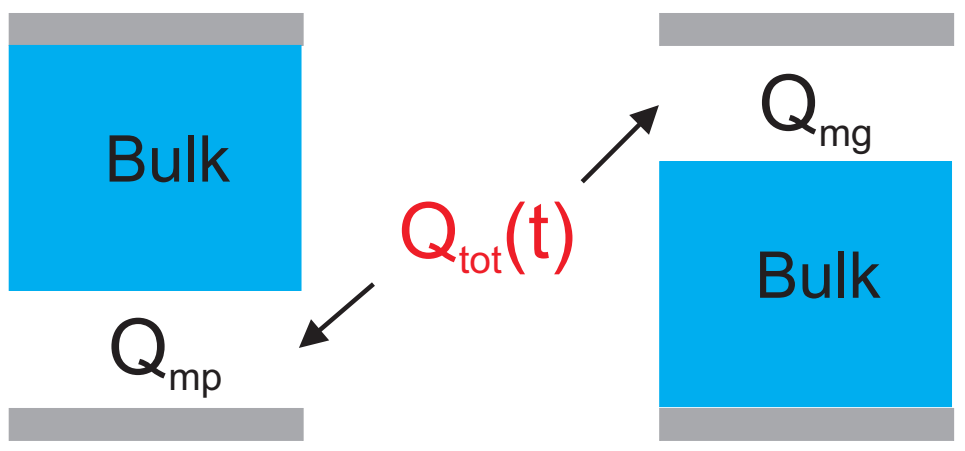

\section{Powered electrode}

\section{$\tilde{\phi}:$}
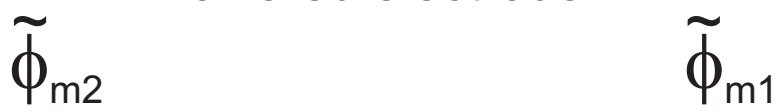

Figure 3. Sketch of the charge distribution in the discharge at the time of minimum $\left(\tilde{\phi}_{m 2}\right)$ and maximum $\left(\tilde{\phi}_{m 1}\right)$ applied voltage. The floating potential is neglected, i.e. the sheath is assumed to collapse completely at both electrodes at least once per RF period.

$Q_{m p}, Q_{m g}$ is the maximum charge in the sheath adjacent to the powered and grounded electrode, respectively. As sketched in figure 3 the charge in the sheath at the powered electrode is maximum, when the minimum voltage, $\tilde{\phi}_{m 2}$, is applied to the discharge. Neglecting the small floating potential of typically a few volts, the sheath at ground collapses completely at this phase and $Q_{t o t}=Q_{m p}$. The charge in the sheath at the grounded electrode is maximum, when the maximum voltage, $\tilde{\phi}_{m 1}$, is applied to the discharge and when the sheath at the powered electrode completely collapses.

In the analytical model of the EAE [19] as well as the Brinkmann sheath model [2023 any dynamics of $Q_{t o t}$ and, consequently, $\sigma_{t o t}$ is neglected, i.e. $Q_{t o t}=Q_{m p}=Q_{m g}$ is assumed. Under this assumption the symmetry parameter resulting from the Brinkmann 
sheath model, which is used in the analytical model of the EAE, $\varepsilon^{\prime}$, depends only on the ratio of the mean ion densities in both sheaths and no longer on the ratio $Q_{m g} / Q_{m p}$ :

$$
\varepsilon^{\prime}=\frac{\bar{n}_{s p}}{\bar{n}_{s g}}
$$

In the following it will be demonstrated that the phase shift $\Delta \theta$ is a consequence of the assumption of $Q_{m g} / Q_{m p}=1$, i.e. the negligence of the charge dynamics.

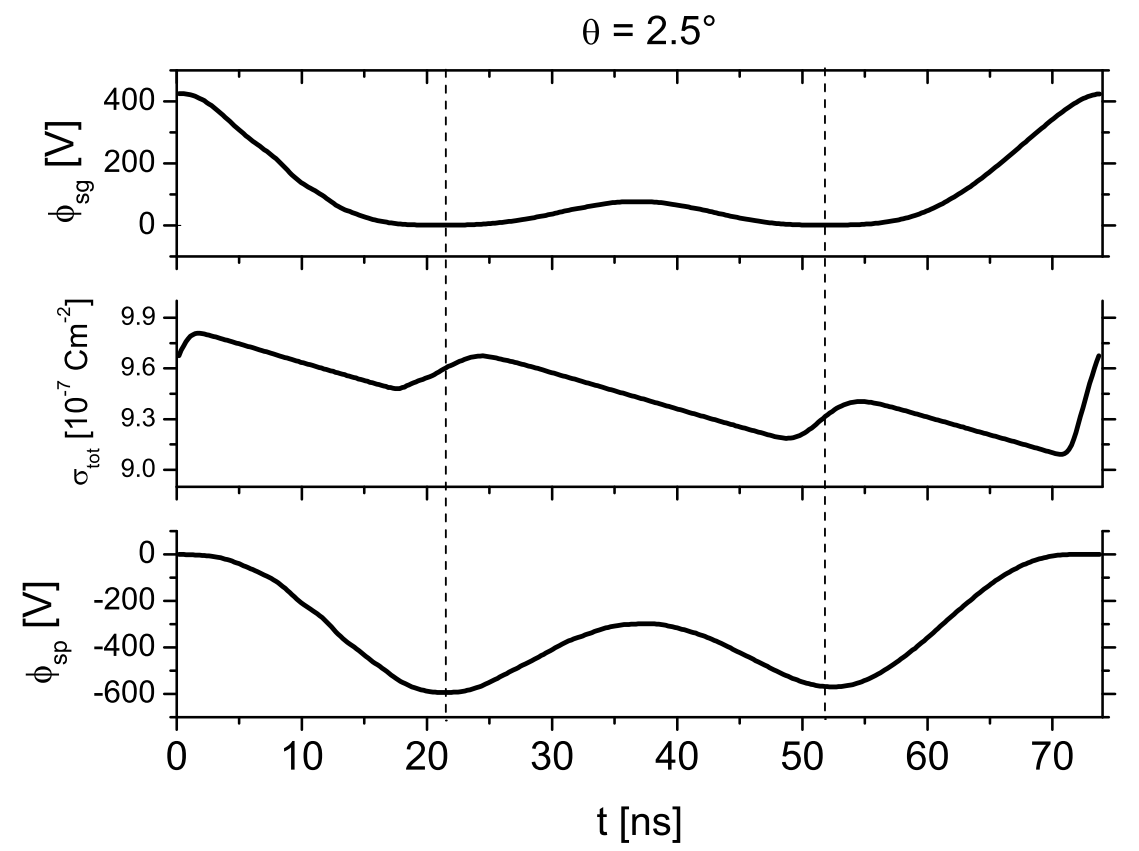

Figure 4. Sheath voltages $\phi_{s g}, \phi_{s p}$ and the total charge in the discharge per area, $\sigma_{t o t}$, as a function of time within one lf period resulting from the PIC simulation of an argon discharge at $\theta=2.5^{\circ}$. The vertical dashed lines indicate the phases of minimum sheath voltage at the grounded electrode within one lf period.

Figures 4 - 6 show the sheath voltages, $\phi_{s g}$ and $\phi_{s p}$, at each electrode (top: Grounded electrode, bottom: Powered electrode) as well as the total charge in the discharge per area, $\sigma_{t o t}$, (middle plot) as a function of time within one low frequency (lf) period at $\theta=2.5^{\circ}, \theta=7.5^{\circ}$, and $\theta=12.5^{\circ}$, respectively (PIC simulation). The vertical dashed lines indicate the phases of minimum sheath voltage at the grounded electrode. At $t=0 \mathrm{~ns}$ the sheath collapses at the powered electrode. The discharge conditions are the same as used in the analytical model and the fluid simulation.

The dynamics of $\sigma_{t o t}$ is similar to the dynamics described by the analytical model: $\sigma_{t o t}$ is positive at all times and fluctuates by about $10 \%$ around its time average value. Ions are continuously lost from the discharge at a constant rate due to the ion fluxes to both electrodes. Only at the short phases of sheath collapse at each of the electrodes electrons are lost and the total positive charge increases again. The only difference between the analytical model described in the previous section and the PIC results shown here is the number of sheath collapses at the grounded electrode and, as a consequence of this, the 


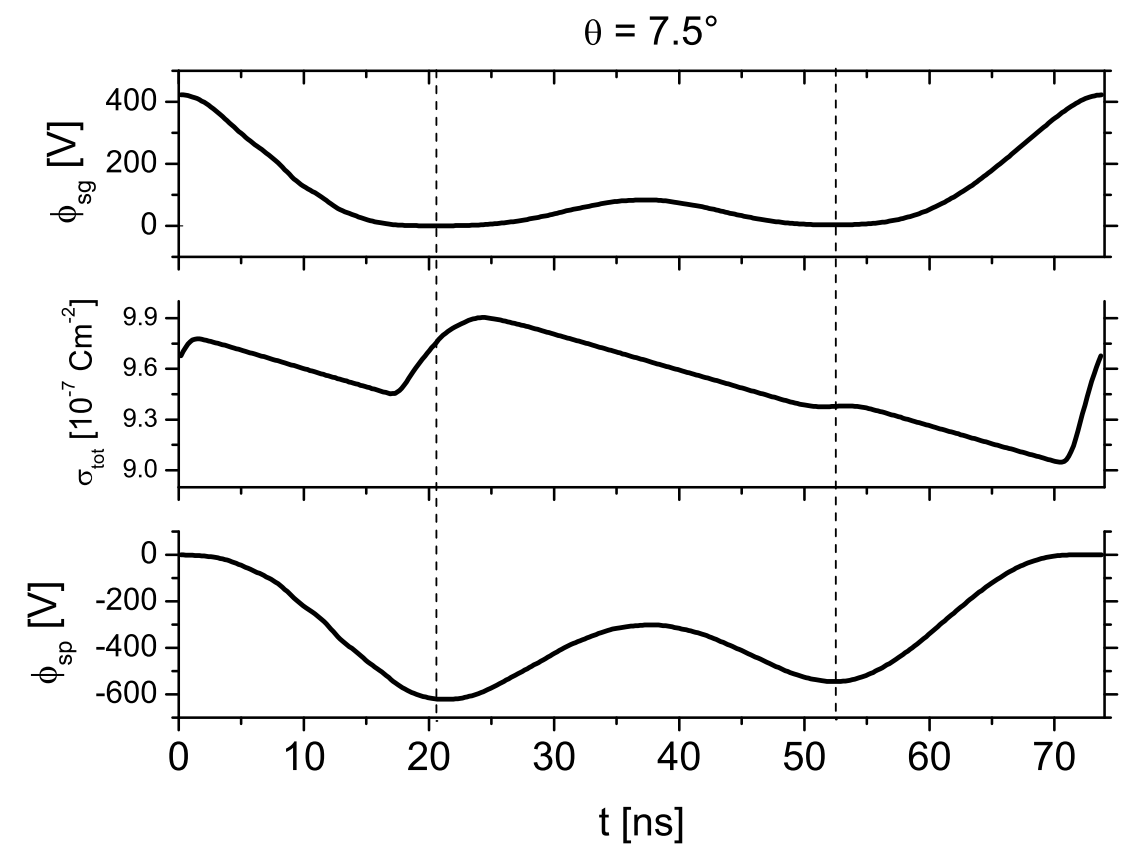

Figure 5. Sheath voltages $\phi_{s g}, \phi_{s p}$ and the total charge in the discharge per area, $\sigma_{t o t}$, as a function of time within one lf period resulting from the PIC simulation of an argon discharge at $\theta=7.5^{\circ}$. The vertical dashed lines indicate the phases of minimum sheath voltage at the grounded electrode within one lf period.

time between two consecutive sheath collapses. Due to the applied voltage waveform the sheath collapses twice at the grounded electrode within one lf period and the time between two consecutive sheath collapses is about $T_{L F} / 3$. Here $T_{L F}$ is the duration of one lf period. However, the analytical model still predicts the fluctuation of $\sigma_{\text {tot }}$ quite accurately.

The sheath dynamics and particularly the total number of sheath collapses at both electrodes are affected by the choice of the phase angle $\theta$. This is shown in figures 4 6: For all phase angles the sheath collapses once at the powered electrode at $t=0 \mathrm{~ns}$. However, the number of sheath collapses at the grounded electrode changes as a function of $\theta$. At $\theta=2.5^{\circ}$ the sheath collapses twice at the grounded electrode at times indicated by the vertical dashed lines in figure 4 . Thus, electrons are lost to the grounded electrode twice per lf period. With increasing $\theta$ the minimum sheath voltage at the phase, when the sheath at ground collapses the second time (second vertical dashed lines in figures 4 - 6), increases until no electrons can cross this potential barrier at $\theta=12.5^{\circ}$ and electrons are only lost to the grounded electrode during the first sheath collapse. Figure 7 shows the minimum voltage drop across the sheath at ground at the phase of the second sheath collapse. The vertical dashed line in figure 7 marks the phase angle of $12.5^{\circ}$, when no electrons reach the grounded electrode anymore at this time within the If period. For $12.5^{\circ}<\theta<77.5^{\circ}, \partial \sigma_{t o t} / \partial t$ remains constant around the second time of minimum sheath voltage at the grounded electrode. 


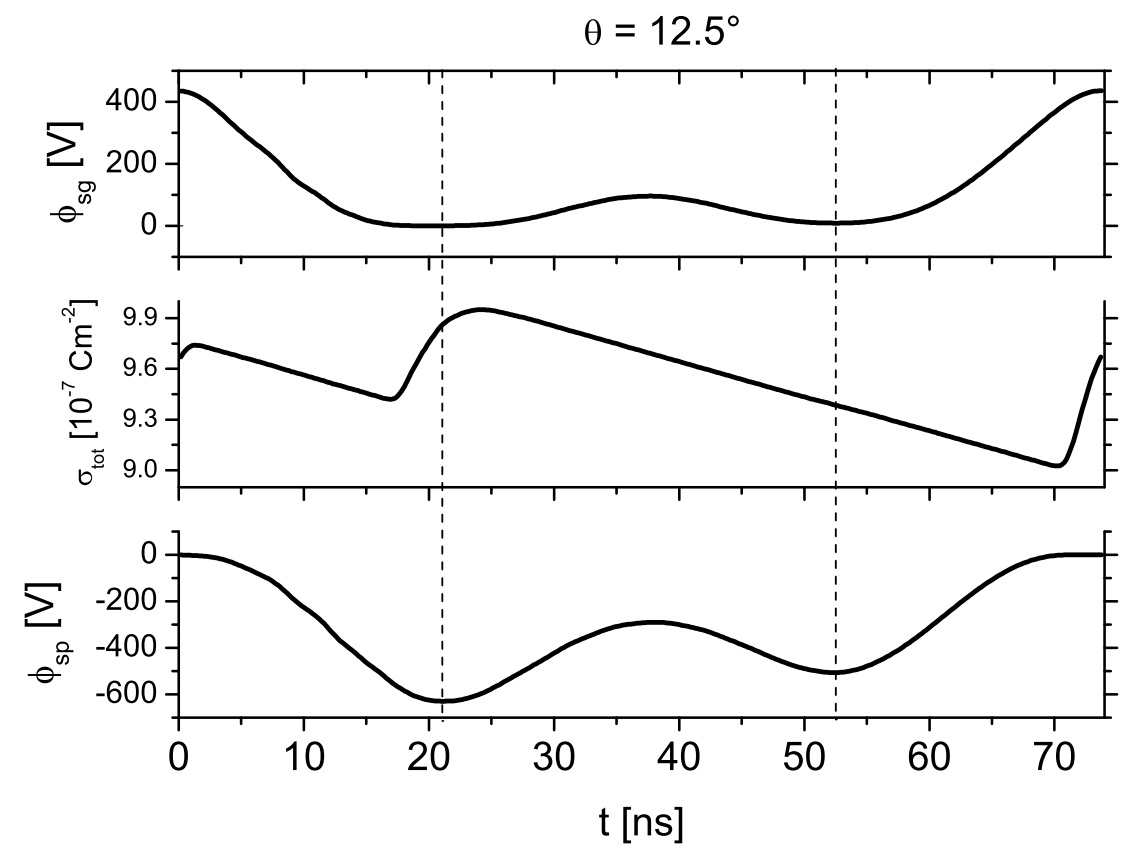

Figure 6. Sheath voltages $\phi_{s g}, \phi_{s p}$ and the total charge in the discharge per area, $\sigma_{t o t}$, as a function of time within one lf period resulting from the PIC simulation of an argon discharge at $\theta=12.5^{\circ}$. The vertical dashed lines indicate the phases of minimum sheath voltage at the grounded electrode within one lf period.

The number of electrons lost to each electrode within one RF period has to compensate the number of ions lost to the same electrode within one RF period. Consequently, if the sheath at ground collapses only once, all electrons required to compensate the ion flux to this electrode will leave the discharge during this one phase of sheath collapse. If the sheath at ground collapses twice, the electron flux to the grounded electrode will be distributed between the two sheath collapses. Consequently, the total charge in the discharge at the phase of the first sheath collapse at ground will be higher, if the sheath at ground collapses only once compared to two sheath collapses. This causes the maximum charge in the sheath at the powered electrode $Q_{m p}$ to increase as a function of $\theta$, while the maximum charge in the sheath at ground $Q_{m g}$ remains approximately constant.

The ratio of $Q_{m g}$ to $Q_{m p}$ is determined from the strongest electric fields at each electrode (PIC simulation) using equations 7 and 8 . Figure 8 shows the square of this ratio as a function of $\theta$. Obviously, the ratio is generally not unity such as assumed in the analytical model of the EAE and the fluid simulation (no dynamics of $\sigma_{t o t}$ ). It decreases as a function of $\theta$ due to the charge dynamics. This decrease particularly affects the calculation of the symmetry parameter $\varepsilon$ (equation 21 ).

Knowing the correct ratio of the maximum charges in both sheaths from figure 8 , the symmetry parameter resulting from the fluid sheath model performed under the same 


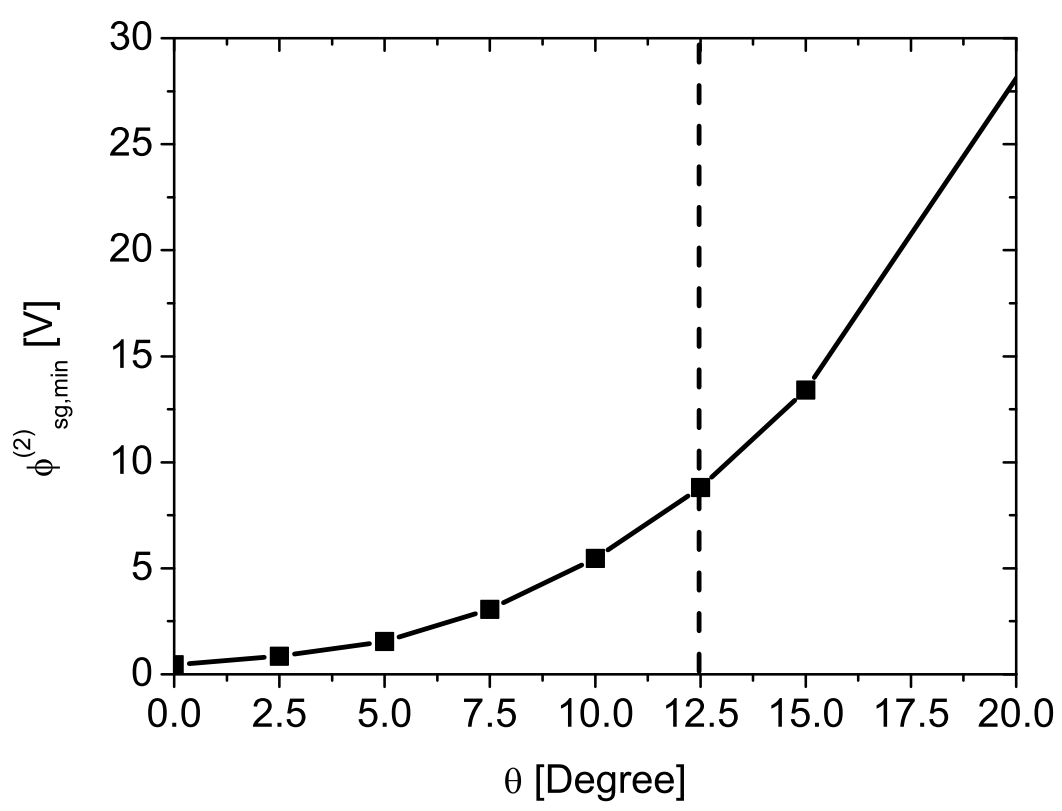

Figure 7. Minimum voltage drop across the sheath at ground at the phase of the second sheath collapse indicated by the second vertical dashed lines in figures $4-6$ as a function of $\theta$. The discharge conditions are the same as in figures $4-6$. The vertical dashed line marks the phase angle of $12.5^{\circ}$, when no electrons reach the grounded electrode anymore at this time within the lf period.

discharge conditions (equation 22 and blue line in figure 9) can be corrected for each $\theta$ :

$$
\varepsilon_{c o r r}=\varepsilon^{\prime} \cdot\left(\frac{Q_{m g}}{Q_{m p}}\right)^{2}
$$

Figure 9 shows the result of this correction. The corrected symmetry parameter reproduces the symmetry parameter resulting directly from the PIC simulation. Obviously, the difference of the symmetry parameters resulting from the fluid sheath model and the PIC simulation is caused by the charge dynamics.

If the symmetry parameter directly resulting from the PIC simulation, which is well approximated by the corrected $\varepsilon$ from the sheath model, is used as an input parameter in the analytical model of the EAE, the DC self bias calculated by the analytical model is essentially identical with the DC self bias resulting from the PIC simulation (see figure 10). In conclusion, the phase shift $\Delta \theta$ between the DC self bias resulting from the analytical model/Brinkmann sheath model and the PIC simulation is caused by the charge dynamics in the discharge, which is affected by the choice of $\theta$ (one or two sheath collapses at ground).

Figure 11 shows the absolute value of the global minimum of the sheath voltage at the powered and grounded electrode as a function of $\theta$, respectively (PIC simulation: $\left.\tilde{\phi}_{0}=100 \mathrm{~V}, 6 \mathrm{~Pa}\right)$. The minimum voltage drop across the sheath at the powered electrode is about $1 \mathrm{~V}$ and essentially does not change as a function of $\theta$. The minimum 


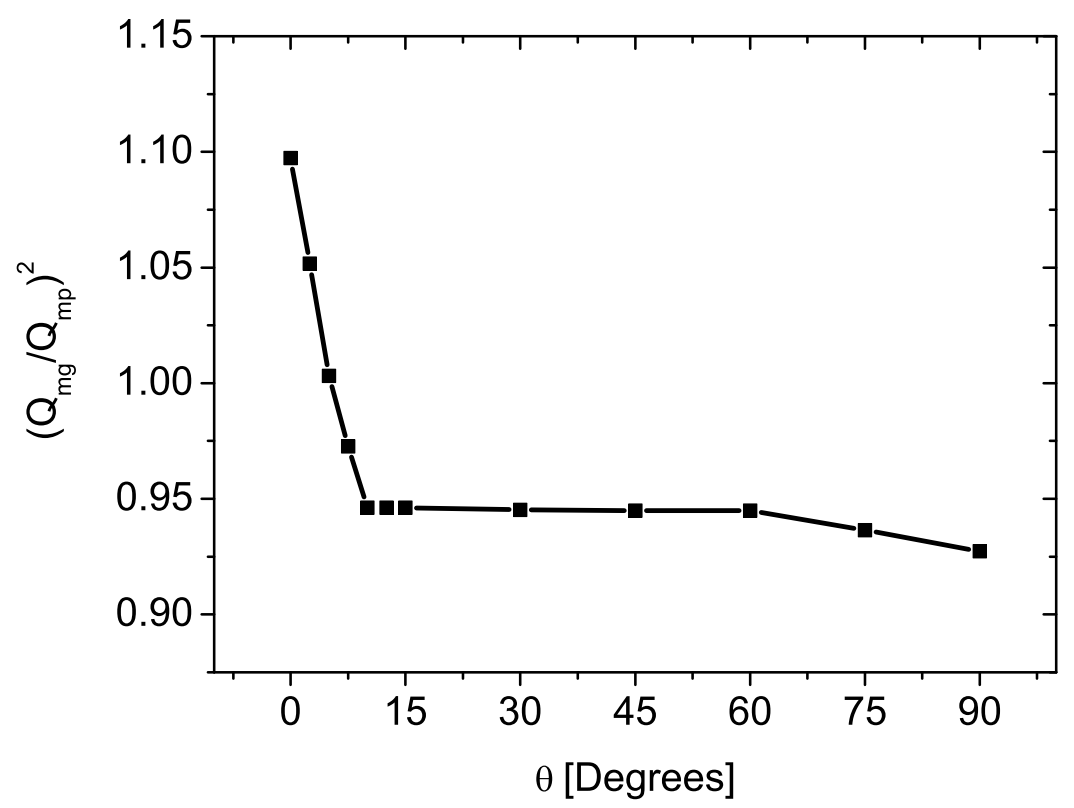

Figure 8. Square of the ratio of the maximum charge in the sheath at ground and the maximum charge in the sheath at the powered electrode as a function of $\theta$ obtained from the PIC simulation.

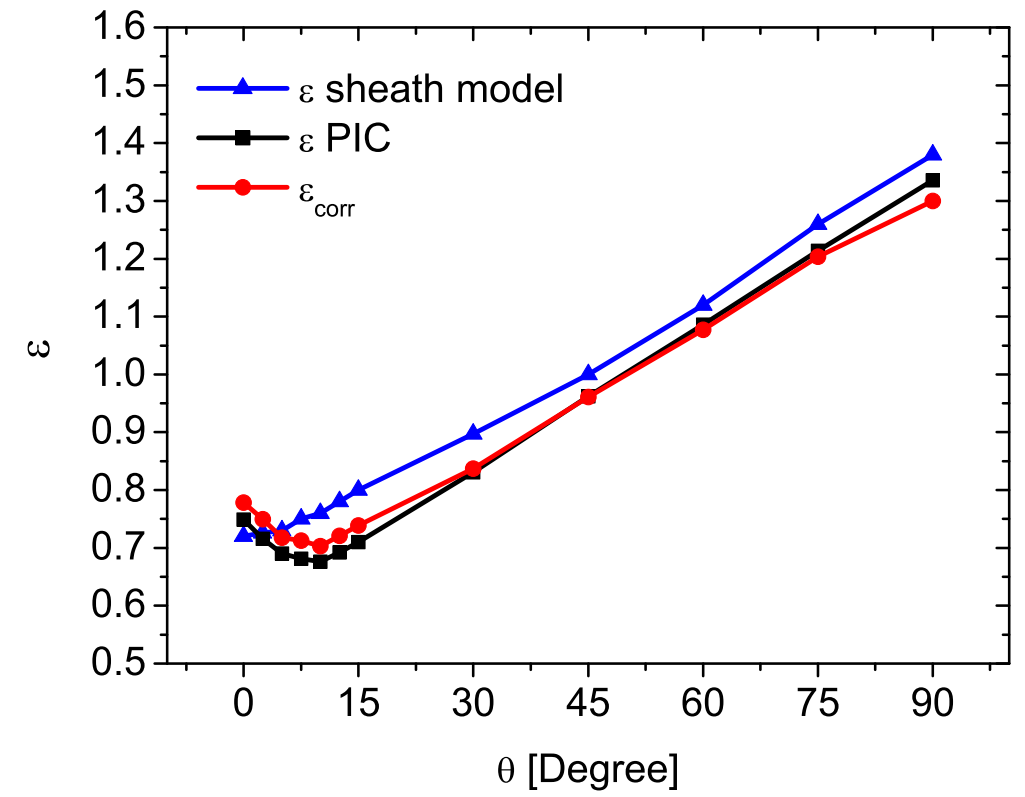

Figure 9. Symmetry parameter resulting from the fluid sheath model (triangles), the PIC simulation (squares), and resulting from equation 23 (circles).

voltage drop across the sheath at the grounded electrode, however, changes as a function of $\theta$ : A maximum of about $4.5 \mathrm{~V}$ is observed at small $\theta$ around $0^{\circ}$. This phenomenon 


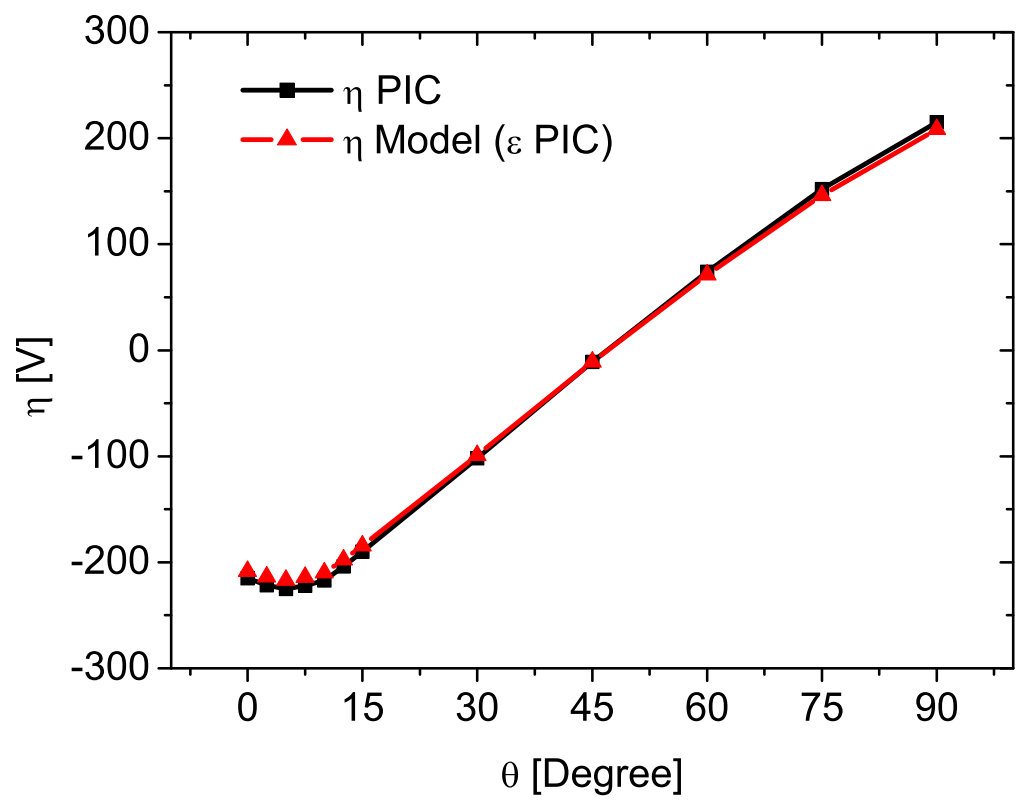

Figure 10. DC self bias $\eta$ calculated by the analytical model using $\varepsilon$ from the PIC simulation as an input parameter.

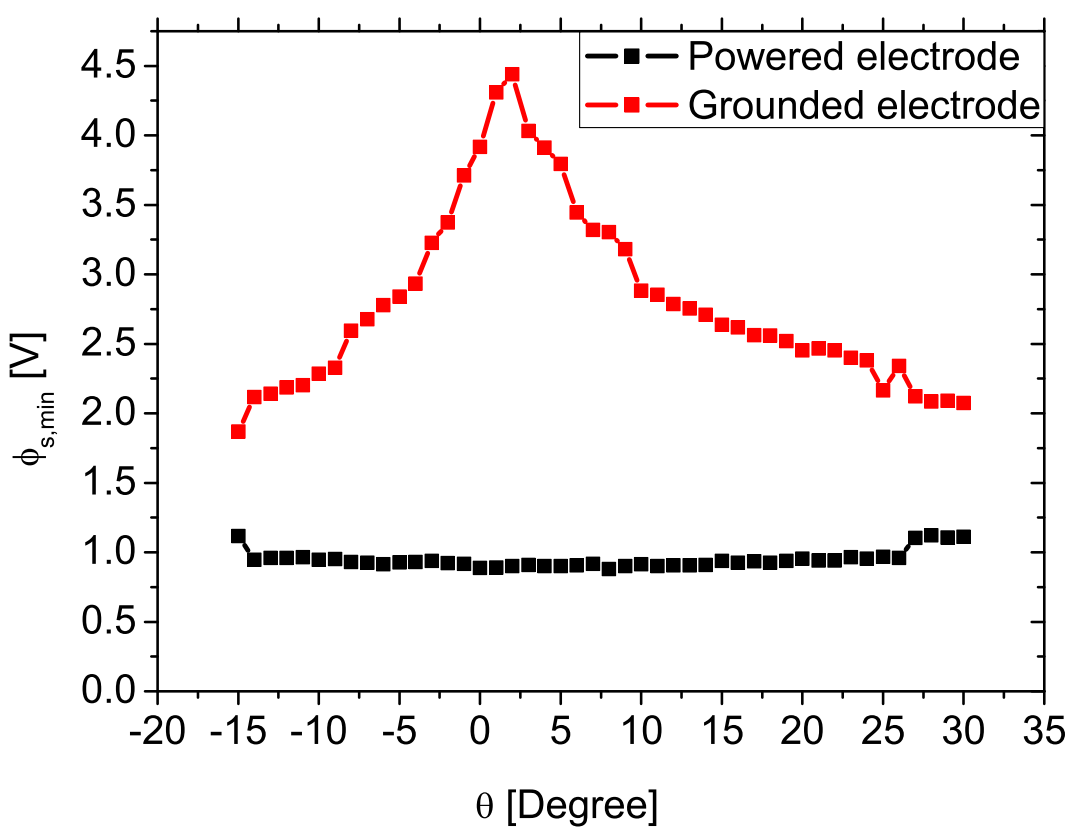

Figure 11. Absolute values of the global minima of the sheath voltages at the powered and grounded electrode as a function of $\theta$ (PIC simulation: $\tilde{\phi}_{0}=100 \mathrm{~V}, 6 \mathrm{~Pa}$ ). 
can be understood based on the understanding of the charge dynamics outlined above: At small values of $\theta$ the sheath adjacent to the grounded electrode collapses twice, while it collapses only once for larger $\theta$. Thus, for small values of $\theta$ the electrons required to compensate the ion flux reach the grounded electrode during a longer time (two sheath collapses) compared to larger values of $\theta$ (one sheath collapse). Consequently, the minimum sheath potential must be higher for small $\theta$ to ensure flux compensation of electron and ions at the grounded electron within one lf period. The minimum voltage drop across the sheath adjacent to the powered electrode remains constant as a function of $\theta$, since this sheath collapses only once within one lf period independent of $\theta$ for $0 \leq \theta \leq 90^{\circ}$.

\subsection{Dynamics of the sheath charges in an electrically asymmetric dual frequency discharge}

In this section the dynamics of the charge densities in each sheath, $\sigma_{p, g}$, in a geometrically symmetric electrically asymmetric dual frequency discharge operated at $13.56 \mathrm{MHz}$ and $27.12 \mathrm{MHz}$ with fixed, but adjustable phase shift $\theta$ between the driving voltages is investigated by a PIC simulation. The discharge is operated in argon at $3 \mathrm{~Pa}$ and an electrode gap of $2.5 \mathrm{~cm}(\alpha=\gamma=0)$. The voltage waveform defined by equation 2 with $\tilde{\phi}_{0}=800 \mathrm{~V}$ is applied to the bottom electrode.

Figure 12 (a) shows the total charge per area, $\sigma_{\text {tot }}$, as a function of time within one lf period at $\theta=7.5^{\circ}$. At this phase angle the strongest DC self bias and the strongest electrical asymmetry are observed. Similar to the results discussed in the previous section the total charge in the discharge is not temporally constant due to the Bohm fluxes of ions to both electrodes, which are compensated by electrons at phases of sheath collapse. At this phase angle $\theta$ almost all electrons required to compensate the ion flux to the grounded electrode leave the discharge during the first sheath collapse at ground. Figure $12(\mathrm{~b})$ shows a phase space plot of $\sigma_{p}$ (solid blue line) and $\sigma_{g}^{\max }-\sigma_{g}$ (dashed red line). $\sigma_{g}^{\max }$ is the maximum charge in the sheath adjacent to the grounded electrode and $\varphi=2 \pi f t$. A phase space plot of $\sigma_{g}^{\max }-\sigma_{g}$ is shown in order to allow a better comparison of the dynamics of both sheaths. The small differences between both curves are caused by the temporal changes of $\sigma_{t o t}$. After the sheath collapse the charge in and the width of the sheaths increase monotonically, while the sheath expansion velocity increases non-monotonically and reaches its maximum value at a relatively small expansion of the sheath. The oscillations in charge phase space are caused by the self excitation of non-linear PSR oscillations of the RF current [4,28,39-53]. This phenomenon is understood based on the voltage balance of a CCRF discharge in proper normalization such as defined in reference [51]:

$$
\eta+\tilde{\phi}=-q^{2}+\varepsilon\left(q_{\mathrm{t}}-q\right)^{2}+\phi_{\mathrm{b}}
$$

here $q$ is the normalized unbalanced charge in the sheath at the powered electrode, $q_{t}$ is the normalized total unbalanced charge in the discharge, and $\phi_{\mathrm{b}}=$ 

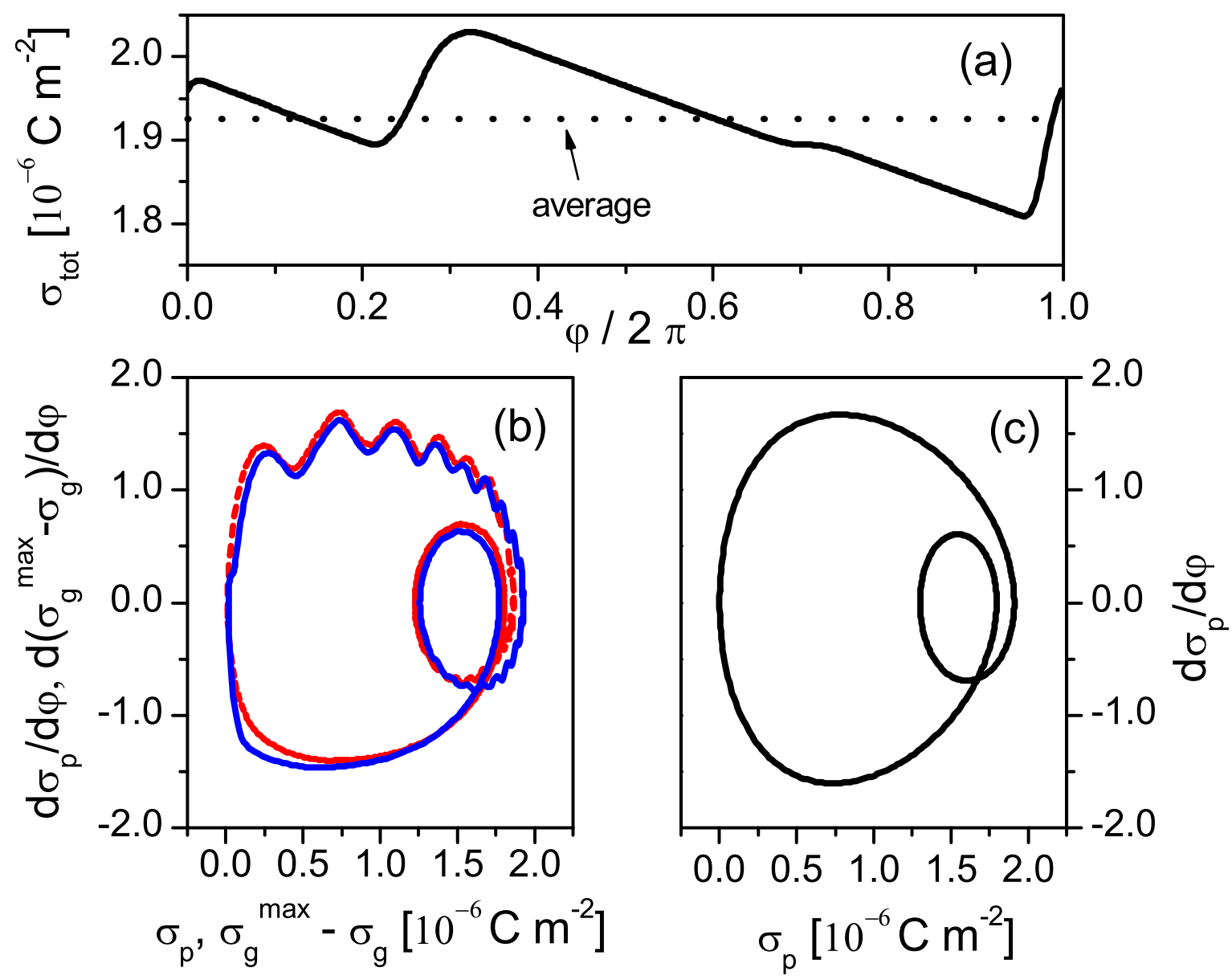

Figure 12. (a) Total net positive charge in the discharge per area as a function of the phase within one lf period. (b) Phase space plot of $\sigma_{p}$ (solid blue line) and $\sigma_{g}^{\text {max }}-\sigma_{g}$ (dashed red line). $\sigma_{g}^{\max }$ is the maximum charge in the sheath adjacent to the grounded electrode and $\varphi=2 \pi f t$. (c) Solution of equation 24 without $\phi_{\mathrm{b}} \cdot \theta=7.5^{\circ}$. In (b) and (c) the time evolution is clockwise.

$-2\left(\partial^{2} q / \partial t^{2}+\kappa \partial q / \partial t\right)$ is the voltage drop across the plasma bulk ( $\kappa$ is a damping constant [51]). Regarding $\phi_{\mathrm{b}}$ the first term represents electron inertia and the second term the resistive part (collisions).

In the PSR model [51] the sheath charge voltage relation is assumed to be quadratic such as verified experimentally before [4]. This is also confirmed by the PIC simulation: Figure 13 shows the charge voltage relation for both sheaths resulting from the PIC simulation under the same discharge conditions as investigated before. A simplified quadratic charge voltage relation resulting from an analytical model of Heil et al. [19] is plotted for comparison (powered sheath), using the ion density directly in front of the 


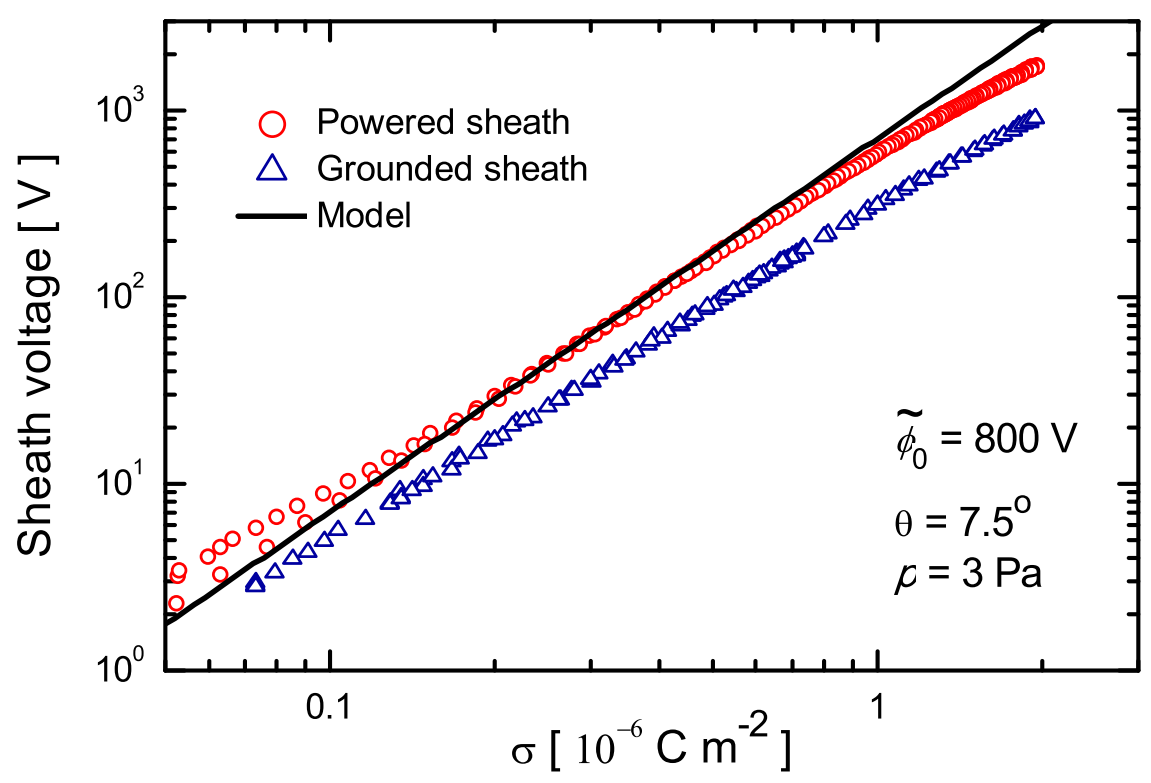

Figure 13. Voltage drops across the sheaths at both electrodes as a function of the charge contained in the sheaths (normalized by the electrode area) at $\theta=7.5^{\circ}$. The solid (black) line shows the result of an analytical model (equation 25, [19]) for the charge-voltage relation of the sheath at the powered electrode using the ion density at the electrode.

electrode as an input parameter:

$$
\phi_{s}(t) \approx-\frac{1}{2 e \varepsilon_{0} \bar{n}_{s} A_{s}^{2}} Q_{s}(t)^{2}
$$

Here $\phi_{s}(t)$ is the time dependent sheath voltage, $\bar{n}_{s}$ is the mean ion density in the sheath approximated by the ion density directly in front of the electrode, $A_{s}$ is the electrode surface area adjacent to the sheath, and $Q_{s}(t)$ is the time dependent uncompensated charge in the sheath. Obviously, the charge voltage relation is quadratic to a good approximation. Slight deviations from the quadratic relation are due to the actual density profile in the sheath. At voltages below about $k T_{\mathrm{e}} / e$ deviations from $q^{2}$ are due to the density profile of the electrons in the sheath.

The non-linear charge voltage relation of the sheath, $q^{2}$, and the inertia term, $\partial^{2} q / \partial t^{2}$, in equation 24 form a non-linear oscillator, which leads to high-frequency harmonics (more than an order of magnitude higher than the driving RF frequency) of the current with large amplitudes, if (i) collisional damping is sufficiently low, i.e. at low pressures (small $\kappa)$, and (ii) the non-linearity $q^{2}$ in equation 24 does not cancel. In case of a conventional geometrically symmetric discharge the electrode areas $A_{\mathrm{p}}, A_{\mathrm{g}}$ and the mean ion densities in the respective sheath $\bar{n}_{\mathrm{sp}}, \bar{n}_{\mathrm{sg}}$ are equal and $\varepsilon=1$. In this case the individual sheaths 
are still non-linear, but the non-linearity cancels for the sum of both sheath voltages and PSR oscillations due to even non-linearities cannot be observed. On the other hand, if the grounded area is infinitely larger than the area of the powered electrode, $\varepsilon$ will approach zero. In this case the non-linearity will not cancel. Clearly this condition is optimum for the generation of PSR oscillations.

The EAE provides a means to achieve $\varepsilon \neq 1$ even in geometrically symmetric discharges so that PSR oscillations become possible [28]. At low pressures the variable DC selfbias generated by the EAE leads to different mean ion densities in both sheaths and, therefore, to $\varepsilon \neq 1$ (see figure 9 ). In this way an asymmetry in the sheaths is created electrically instead of geometrically.

No vortices are generated by the PSR effect. The single big vortex is caused by the applied voltage waveform. Similarities to the sheath dynamics in standard dualfrequency discharges operated at substantially different frequencies are obvious $[3,13$, 14]. Figure 12 (c) shows the solution of equation 24 without the resistive/inductive term $\phi_{\mathrm{b}}$. Obviously, this term and, therefore, electron inertia is essential for the generation of high frequency self excited PSR oscillations.

\section{Conclusions}

An analytical model to describe the charge dynamics in CCRF discharges has been developed. In contrast to the assumption of a temporally constant total uncompensated charge in the discharge made in several models of CCRF discharges it predicts a modulation of $\sigma_{t o t}$ by about $10 \%$ around its time average value within one lf period. This modulation is caused by the constant ion flux to both electrodes and the strongly time dependent electron flux to the electrodes, that compensates the ion flux to each electrode within one RF period at phases of sheath collapse. The constant ion fluxes to the electrodes lead to a constant loss rate of positive charge from the discharge, while the electron losses lead to an increase of the total uncompensated net positive charge at phases of sheath collapse.

The dynamics of $\sigma_{t o t}$ in a geometrically symmetric, but electrically asymmetric dual frequency CCRF discharge operated at $13.56 \mathrm{MHz}$ and $27.12 \mathrm{MHz}$ with variable phase shift $\theta$ between the driving voltages has been investigated by a PIC simulation. A variable $\mathrm{DC}$ self bias is generated as a function of $\theta$ via the EAE. Charge dynamics similar to the ones predicted by the analytical model are observed. A phase shift $\Delta \theta \approx 8^{\circ}$ between the DC self bias as a function of $\theta$ resulting from the PIC simulation on one side and previous models of the EAE on the other side is found. In these models of the EAE any charge dynamics is neglected. It is demonstrated that this negligence causes the observed phase shift. The charge dynamics is found to be affected by the choice of $\theta$ (one or two sheath collapses at ground).

Finally, the total uncompensated charge in the discharge is split into the uncompensated charges in each sheath. The dynamics of the sheath charges is investigated in charge phase space and the self-excitation of non-linear PSR oscillations of the RF current is 
observed at low pressures. This phenomenon is understood based on a previous PSR model and the fact, that the EAE causes a variable (electrical) discharge asymmetry required for the self-excitation of PSR oscillations.

\section{Acknowledgments}

This work has been funded by the DFG through GRK 1051, the Ruhr-University Research Department Plasma, the Ruhr University Research School, and the Hungarian Scientific Research Fund through grant OTKA-K-77653.

\section{References}

[1] Lieberman M A and Lichtenberg A J 2005, Principles of Plasma Discharges and Materials Processing, 2nd. ed., Wiley Interscience, NJ: Wiley

[2] Schulze J 2009 PhD thesis, Ruhr University Bochum, Germany http://www-brs.ub.ruhr-unibochum.de/netahtml/HSS/Diss/SchulzeFelixJulian/diss.pdf

[3] Schulze J, Donkó Z, Luggenhölscher D, and Czarnetzki U 2009 Plasma Sources Sci. Technol. 18 034011

[4] Schulze J, Heil B G, Luggenhölscher D, Brinkmann R P, and Czarnetzki U 2008 J. Phys. D 41 195212

[5] Turner M M 2009 J. Phys. D 42194008

[6] Lieberman M A 1988 IEEE Trans. on Plasma Sci. 16 No. 6638

[7] Lieberman M A and Godyak V A 1998 IEEE Trans. on Plasma Sci. 26 No. 3955

[8] Surendra M and Graves D B 1991 Phys. Rev. Lett. 661469

[9] Turner M M 1995 Phys. Rev. Lett. 751312

[10] Gozadinos G, Turner M M and Vender D 2001 Phys. Rev. Lett. 87135004

[11] Kaganovich I D 2002 Phys. Rev. Lett. 89265006

[12] Kaganovich I D, Polomarov O V and Theodosioue C E 2006 IEEE Trans. on Plasma Sci. 34 No. 3696

[13] Gans T, Schulze J, O'Connell D, Czarnetzki U, Faulkner R, Ellingboe A R, and Turner M M 2006 Appl. Phys. Lett. 89261502

[14] Schulze J, Gans T, O'Connell D, Czarnetzki U, Ellingboe A R, and Turner M M 2007 J. Phys. D 407008

[15] Booth J P, Curley G, Marić D, and Chabert P 2010 Plasma Sources Sci. Technol. 19015005

[16] Wild C and Koidl P 1990 J. Appl. Phys. 692909

[17] Field D, Klemperer D F, May P W, Song Y P 1991 J. Appl. Phys. 7082

[18] Flender U and Wiesemann K 1994 J. Phys. D 27509

[19] Heil B G, Czarnetzki U, Brinkmann R P and Mussenbrock T 2008 J. Phys. D 41165202

[20] Brinkmann R P 2007 J. Appl. Phys. 102093303

[21] Heil BG, Brinkmann RP, Czarnetzki U 2008 J. Phys. D 41225208

[22] Brinkmann R P 2009 J. Phys. D 42194009

[23] Heil BG, Brinkmann RP, Czarnetzki U 2009 J. Phys. D 42085205

[24] Heil B G, Schulze J, Mussenbrock T, Brinkmann R P and Czarnetzki U 2008 IEEE Trans. on Plasma Sci. 361404

[25] Czarnetzki U, Heil B G, Schulze J, Donkó Z, Mussenbrock T and Brinkmann R P 2009 IOP Conf. Series 162012010

[26] Donkó Z, Schulze J, Heil B G, and Czarnetzki U 2008 J. Phys. D 42025205

[27] Schulze J, Schüngel E, Donkó Z and Czarnetzki U 2009 J. Phys. D FTC 42092005

[28] Donkó Z, Schulze J, Czarnetzki U, Luggenhölscher D 2009 Appl. Phys. Lett. 94131501 
[29] Longo S, Diomede P 2009 Plasma Processes and Polymers 6370

[30] Schulze J, Schüngel E, Donkó Z, and Czarnetzki U 2009 J. Appl. Phys. 106063307

[31] Schulze J, Schüngel E, Donkó Z, and Czarnetzki U 2009 Plasma Sources Sci. Technol. submitted for publication

[32] Phelps A V, Petrović Z Lj 1999 Plasma Sources Sci. Technol. 8 R21

[33] Phelps A V 1994 J. Appl. Phys. 76747

[34] Phelps A V http://jilawww.colorado.edu/ avp/collision_data/ unpublished

[35] Schulze J, Donkó Z, Heil B G, Luggenhölscher D, Mussenbrock T, Brinkmann R P and Czarnetzki U 2008 J. Phys. D 41105214

[36] Donkó Z and Petrović Z Lj 2006 J. Phys.: Conf. Series 86012011

[37] Donkó Z, Petrovic Z L 2006 Jpn. J. Appl. Phys. Part 1458151

[38] Godyak V A, Piejak R B, and Alexandrovich B M 1992 Plasma Sources Sci. Techn. 136

[39] Schneider F 1954 Angew. Math. Phys. 6456

[40] Taillet J 1969 Osaka Math. J. 37423

[41] Godyak V A and Popov O A 1979 Sov. J. Plasma Phys. 5400

[42] Annaratone B M, Ku V P T, and Allen J E 1995 J. Appl. Phys. 775455

[43] Qiu W D, Bowers K J, and Birdsall C K 2003 Plasma Sources Sci. Technol. 1257

[44] Klick M 1996 J. Appl. Phys. 793445

[45] Mussenbrock T, Brinkmann R P, Lieberman M A, Lichtenberg A J, and Kawamura E 2008 Phys. Rev. Lett 101085004

[46] Mussenbrock T, and Brinkmann R P 2006 Appl. Phys. Lett. 88151503

[47] Mussenbrock T, Ziegler D, and Brinkmann R P 2006 Phys. Plasmas 13083501

[48] Mussenbrock T and Brinkmann R P 2007 Plasma Sources Sci. Techn. 16377

[49] Mussenbrock T, Brinkmann R P, Lieberman M A, Lichtenberg A J, and Kawamura E 2008 Phys. Plasmas 15063505

[50] Ziegler D, Mussenbrock T, and Brinkmann R P 2008 Plasma Sources Sci. Technol. 17045011

[51] Czarnetzki U, Mussenbrock T, and Brinkmann R P 2006 Phys. Plasmas 13123503

[52] Schulze J, Heil B G, Luggenhölscher D, and Czarnetzki U 2008 IEEE Trans. Plasma Sci. 361400

[53] Schulze J, Kampschulte T, Luggenhölscher D, and Czarnetzki U 2007 Journal of Phys.: Conf. Series $\mathbf{8 6} 012010$ 